Jeff Terryberry*, Jani Tuomi, Subo Perampalam, Russ Peloquin, Eric Brouwer, Detlef Schuppan ${ }^{\mathrm{a}}$ and Stefano Guandalini ${ }^{\mathrm{a}}$

\title{
Diagnostic accuracy of a fully automated multiplex celiac disease antibody panel for serum and plasma
}

https://doi.org/10.1515/cclm-2019-0088

Received December 21, 2018; accepted February 20, 2019; previously published online March 23, 2019

\section{Abstract}

Background: An automated multiplex platform using capillary blood can promote greater throughput and more comprehensive studies in celiac disease (CD). Diagnostic accuracy should be improved using likelihood ratios for the post-test probability of ruling-in disease.

Methods: The Ig_plex ${ }^{\mathrm{TM}}$ Celiac Disease Panel on the sqidlite $^{\mathrm{TM}}$ automated platform measured IgA and IgG antibodies to tTG and DGP in $\mathrm{n}=224 \mathrm{CD}$ serum or plasma samples. Diagnostic accuracy metrics were applied to the combined multiplex test results for several CD populations and compared to conventional single antibody ELISA tests.

Results: With multiple positive antibody results, the posttest probability for ruling-in untreated and treated CD increased to over $90 \%$. The number of samples positive for more than one antibody also increased in untreated CD to $\geq 90 \%$. Measurement of all four CD antibodies generate cut-off dependent accuracy profiles that can monitor response to treatment with the gluten-free diet (GFD). Higher positive tTG and DGP antibodies are seen more frequently in confirmed CD without (81\%-94\%) than with GFD treatment (44\%-64\%). In CD lacking biopsy confirmation,

adeflef Schuppan and Stefano Guandalini contributed equally to the manuscript.

*Corresponding author: Jeff Terryberry, SQI Diagnostics Systems Inc., 36 Meteor Dr. Toronto, ON M9W 1A4, Canada,

Phone:+416-674-9500, E-mail: jterryberry@sqidiagnostics.com Jani Tuomi: Microdrop LLC., Houston, TX, USA

Subo Perampalam, Russ Peloquin and Eric Brouwer: SQI Diagnostics Systems Inc., Toronto, ON, Canada

Detlef Schuppan: Division of Gastroenterology, Beth Israel Deaconess Medical Center, Harvard Medical School, Boston, MA, USA; and Institute of Translational Immunology, University Medical Center, Johannes-Gutenberg University, Mainz, Germany Stefano Guandalini: Department of Pediatrics, Section of Gastroenterology, Hepatology and Nutrition, University of Chicago Celiac Disease Center-Comer Children's Hospital, Chicago, IL, USA overall agreement of plasma to serum was $\geq 98 \%$ for all antibodies, and $100 \%$ for venous to capillary plasma.

Conclusions: The Ig_plex Celiac Disease Panel increases the likelihood of confirming CD based on the post-test probability of disease results for multi-reactive markers. Specific positivity profiles and cut-off intervals can be used to monitor GFD treatment and likely disease progression. Using serum, venous and capillary plasma yield comparable and accurate results.

Keywords: celiac disease; diagnostic accuracy; gluten; likelihood ratio; multiplexing.

\section{Introduction}

Celiac disease (CD), wheat allergy (WA) and non-celiac wheat (gluten) sensitivity (NCWS, NCGS) represent different wheat-induced inflammatory disorders, for practical reasons here termed gluten-related disorders (GRD). GRDs, with an estimated global prevalence of up to $5 \%$, have received increasing attention as a clinically relevant spectrum of diseases in recent evaluations of nomenclature and classification [1-4]. Epidemiological studies estimate a worldwide prevalence of CD of approximately $1 \%$, with an additional $1 \%$ of CD cases undiagnosed, indicating a need for improvements in screening for $\mathrm{CD}$ in the general population $[5,6]$. In addition, the need to rule out CD before labeling a patient with NCGS is necessary prior to initiating a gluten-free diet (GFD). Further, a portion of patients labeled with NCGS may not be reacting to gluten, but to fermentable oligo-, di-, mono-saccharides and polyols or wheat amylase trypsin inhibitors, showing only a placebo/nocebo effect. It is thus evident that before administering a GFD, accurate diagnostic criteria and markers are needed to characterize people with CD or other GRD as this leads to different nutritional and treatment strategies [2, 7-11].

Gluten-containing cereal prolamins, such as gliadin in wheat, secalin in rye and hordein in barley, have a high number of proteolysis-resistant sequences, resulting in the persistence of larger peptides in the gut. These peptides 
can then trigger an innate immune response or activate small-bowel mucosal CD4+ T cells in patients who carry the necessary genetic predisposition for $\mathrm{CD}$, the antigen presenting molecules HLA-DQ2 and/or -DQ8. Intestinal tissue transglutaminase (tTG), a ubiquitous enzyme that is released from intestinal cells during mechanical or cellular stress, has been identified as the autoantigen of $\mathrm{CD}$, against which the patients' IgA and IgG antibodies, previously described as endomysial autoantibodies, are directed. Importantly, tTG crosslinks and/or deamidates the partly digested gluten peptides in the intestinal lamina propria and increases their immunogenic potential [12, 13]. The importance of antibody detection to the diagnosis of CD is well recognized. Currently, tTG ELISA tests are used as assays to diagnose CD, and TTG-IgA is widely recommended as the most reliable screening test. However, the clinical performance of sensitivity and specificity may vary depending on the quality of the $\mathrm{TTG}$ antigen and the antibody isotype detected [11, 14, 15].

Selective deamidation of gliadins by tTG also increases circulating antibody levels to gliadin peptides in celiac patients. Such serum deamidated gliadin peptide (DGP) antibodies have been proven to be additional indicators of CD. Testing for DGP IgA and IgG antibodies, along with the IgA and IgG tTG antibodies, is recommended in children $\leq 2$ years old, and in patients with serum IgA levels $<0.2 \mathrm{~g} / \mathrm{L}$ where tTG IgA and DGP IgA are likely to yield false-negative results [15-17].

Diagnostic accuracy studies using likelihood ratios (LR), post-test probability of disease and positive predictive value (PPV) have received increased focus as a means to supplement the clinical interpretation of multiple diagnostic test strategies. Multiplexing the known celiac antigen immunoassays increases the diagnostic accuracy of CD detection with multiple LR probability estimates. However, previous studies have only looked at various combinations of two or three CD-related antibodies and reported on associated PPV or LR values [18-23].

The current study comprehensively integrates, for the first time, the diagnostic accuracy values of LR and other metrics for all four CD-related antibodies simultaneously and demonstrates the increased post-test probability of having celiac based on multiple validated Ig_plex test results. Further, this study explores the Ig_plex test's ability to monitor response to treatment and serologically differentiate $\mathrm{CD}$ with and without biopsy-confirmation. In addition, this study compares multiple sample matrices to support the use of plasma, including finger-stick capillaryderived plasma, to increase the accessibility of patient sampling. Previously, finger-stick capillary blood samples have only been used in various point-of-care assays. Capillary blood-derived plasma has now been expanded for use in the automated Ig_plex Celiac Disease Panel multiplexing assay to detect and monitor CD [24-27].

\section{Materials and methods}

\section{Subject population}

Samples from a total of 224 well-characterized subjects (Table 1) were evaluated according to published criteria and Standards for Reporting of Diagnostic Accuracy Studies guidelines. Subject demographics, gastrointestinal symptoms, diagnostic criteria, treatment duration and laboratory testing methods were recorded [1, 9, 28-30]. The study was carried out in accordance with The Code of Ethics of the World Medical Association (Declaration of Helsinki) for experiments involving humans with subject consent obtained for use of samples.

Subject samples were obtained from academic collaborators and commercial sources with defined eligibility criteria. Clinically defined sera of patients with biopsy-confirmed (Marsh III) active or treated CD were collected from the Department of Pediatric Gastroenterology and Nutrition at the Massachusetts General Hospital (MGH) for Children in Boston and the Celiac Center at Beth Israel

Table 1: Classification and demographics of $n=224$ subject samples evaluated for diagnostic accuracy.

\begin{tabular}{|c|c|c|c|c|c|c|c|c|c|}
\hline \multirow[t]{2}{*}{ Group } & \multirow{2}{*}{\multicolumn{2}{|c|}{ Subject classification }} & \multirow{2}{*}{$\begin{array}{l}\text { Sample } \\
\text { matrix/pairing }\end{array}$} & \multirow{2}{*}{$\begin{array}{r}\text { Number of } \\
\text { samples }\end{array}$} & \multicolumn{2}{|c|}{ Gender } & \multicolumn{2}{|c|}{ Age group } & \multirow{2}{*}{$\begin{array}{l}\text { Criteria/definition } \\
{[1,6-9,28]}\end{array}$} \\
\hline & & & & & $M$ & $\mathbf{F}$ & Pediatric & Adult & \\
\hline 1 & Confirmed CD with & GFD ( $\geq 1$ month) & Serum & 52 & 11 & 41 & 7 & 45 & Marsh III \\
\hline 2 & Marsh III biopsy criteria & Not yet GFD treated & Serum & 31 & 6 & 25 & 5 & 26 & Marsh III \\
\hline 3 & \multirow{2}{*}{\multicolumn{2}{|c|}{$\begin{array}{l}\text { CD without confirmation by Marsh III biopsy } \\
\text { (includes potential and classical CD) }\end{array}$}} & Serum & 20 & 8 & 12 & 5 & 15 & Classical \\
\hline 4 & & & Plasma & 40 & 13 & 27 & 3 & 37 & $\begin{array}{l}\text { Classical }(\mathrm{n}=37) \\
\text { potential }(\mathrm{n}=3)\end{array}$ \\
\hline 5 & Wheat allergy & & Plasma & 20 & 14 & 6 & 12 & 8 & $\begin{array}{l}\text { Oral food challenge } \\
\text { and skin prick }\end{array}$ \\
\hline 6 & Normal & & Plasma & 61 & 39 & 22 & 6 & 55 & Healthy donor \\
\hline
\end{tabular}

CD, celiac disease; GFD, gluten-free diet. 
Deaconess Medical Center (BIDMC), Boston, MA. Samples were collected between 2011 and 2014, aliquoted and frozen at $-80^{\circ} \mathrm{C}$. Fresh freezer aliquots experiencing no more than two freeze/thaw cycles were used in the study.

The Oslo definition of classical CD describes the presence of a gluten-induced enteropathy presenting with diarrhea, malnutrition or a malabsorption syndrome (indicated by weight loss, steatorrhea and edema secondary to hypoalbuminemia). The term potential CD is used for individuals with normal small intestinal mucosa who are at increased risk of developing $\mathrm{CD}$ as indicated by positive $\mathrm{CD}$ serology $[1,8]$.

In this study, serum and plasma (EDTA) samples from CD patients that did not meet the Marsh III histology criteria for biopsyconfirmed CD, included mostly classical and several potential CD [1, 28]. The uniformly-defined classical CD samples and three cases of potential CD were labeled as $\mathrm{CD}$ without biopsy confirmation and collected from commercial serum banking enrollment programs including Access Biologics (Vista, CA, USA), SeraCare (Milford, MA, USA), in.vent Diagnostica (Berlin, Germany) and Cleveland Clinic (CC, Cleveland, OH, USA) and stored under the same conditions as the confirmed CD samples. In addition, prospective sample collections of CD samples were initiated (May-Oct 2018) to obtain additional $(\mathrm{n}=49)$ paired serum-plasma samples and paired venous-capillary plasma samples (Table 2) from BioIVT (Westbury, NY, USA) and DxBiosamples (San Diego, CA, USA). Capillary samples were obtained by finger-prick using the Microvette 100 EDTA plasma collection tube (Thermo Fisher Scientific Inc., Portage, MI, USA). WA subjects were obtained from AbBaltis (Sittingbourne, UK) and BioIVT. WA subjects were defined as individuals who had both positive results at wheat allergen extract testing either by serum specific IgE or skin prick tests and had shown clinical reaction at the food challenge test [6, 7]. Individual normal human plasma samples were collected from BioIVT.

\section{Testing methods}

Automated Ig_plex ${ }^{\mathrm{TM}}$ Celiac Disease Panel: The Ig_plex Celiac Disease Panel (SQI Diagnostics, Toronto, ON, Canada) quantitatively measures the presence of both IgA and IgG autoantibodies to tTG and DGP antigens for a single sample in a multiplex assay format on the fully automated sqidlite platform, and offers the ability to add additional antigens or allergens to the printed microarray to increase the differential diagnostic potential of the panel. Dual-layer multiplexing at the capture and detector level facilitates both antigen screening and autoantibody isotyping. Antigen microarrays were non-contact printed by piezoelectric dispensing on covalent-binding epoxy-silane derivatized glass 96-well plates. Recombinant tTG (baculovirus/Sf9 insect cell expressed) and DGP peptides representing immunodominant epitopes from $\alpha$ - and $\gamma$-gliadins linked to a protein carrier are printed in multiple replicate spots to ensure precise within-well measurements. The plate is loaded with standard, controls and samples with a primary incubation of $75 \mathrm{~min}$ at $500 \mathrm{rpm}$ shaker speed. Antibody binding is detected after washing (BioTek, Winooski, VT, USA) the plate by secondary anti-IgA and anti-IgG antibodies labeled with fluorescent dyes which are incubated for another $45 \mathrm{~min}$, washed and scanned (Sensovation AG, Radolfzell, Germany).

Sqidlite $^{\mathrm{TM}}$ automated microarray system: The Ig_plex Celiac Disease Panel was run on the sqidlite automated microarray. The sqidlite system is a multiplex immunoassay platform that automates the assay process for diluting and running samples, standards, controls, assay plate loading, incubating, washing and drying for each individual sample. Once the assay's immunochemical reactions have completed, the sqidlite system performs a three-color fluorescent scan of each well in the microarray, analyzes the data and generates a report containing results for all assay markers. The sqidlite system also includes internal quality checks in the data analysis.

Manual ELISA kits: For method comparison studies, FDA-approved ELISA kits were used for detecting IgA and IgG antibodies to tTG and DGP. ELISA testing conducted at SQI Diagnostics employed the following assays from Inova Diagnostics, Inc. (San Diego, CA, USA): Quanta Lite tTG IgG Cat\# 708755 Lot\# 042287; Quanta Lite tTG IgA Cat\# 708760 Lot\# 043755; Quanta Lite DGP IgG Cat\# 704520 Lot\# 042636; Quanta Lite DGP IgA Cat\# 704525 Lot\# 043751. The assays were performed as specified in the kit's package inserts.

\section{Analytical precision}

Analytical precision was measured using $\%$ coefficient of variation $(\% \mathrm{CV})$ with acceptable levels of variation being $\leq 15 \% \mathrm{CV}$. Precision acceptance criteria were based on FDA and WHO guidelines for immunoassays and bioanalytical methods [31, 32]. Intra-assay (within-run) precision was determined with two positive control (PC)

Table 2: Classification and demographics of $n=49$ subject samples evaluated for matrix equivalence studies.

\begin{tabular}{|c|c|c|c|c|c|c|c|c|}
\hline \multirow[t]{2}{*}{ Group } & \multirow[t]{2}{*}{ Subject classification } & \multirow[t]{2}{*}{ Sample matrix/pairing } & \multirow[t]{2}{*}{ Number of samples } & \multicolumn{2}{|c|}{ Gender } & \multicolumn{2}{|c|}{ Age group } & \multirow{2}{*}{$\begin{array}{l}\text { Criteria/definition } \\
{[1,6-9,28]}\end{array}$} \\
\hline & & & & $M$ & $\mathbf{F}$ & Pediatric & Adult & \\
\hline 7 & $\begin{array}{l}\text { CD without confirmation } \\
\text { by Marsh III biopsy }\end{array}$ & $\begin{array}{l}\text { Serum/plasma matched } \\
\text { samples }\end{array}$ & 34 & 8 & 26 & 1 & 33 & Classical \\
\hline 8 & & $\begin{array}{l}\text { Venous/capillary plasma } \\
\text { matched samples }\end{array}$ & 13 (subset of group 7) & 4 & 9 & 1 & 12 & Classical \\
\hline 9 & Wheat allergy & $\begin{array}{l}\text { Serum/plasma matched } \\
\text { samples }\end{array}$ & 5 & 5 & 0 & 4 & 1 & $\begin{array}{l}\text { Oral food challenge } \\
\text { and skin prick }\end{array}$ \\
\hline 10 & Normal & $\begin{array}{l}\text { Serum/venous plasma/ } \\
\text { capillary plasma matched } \\
\text { samples }\end{array}$ & 10 & 6 & 4 & 1 & 9 & Healthy donor \\
\hline
\end{tabular}

$\mathrm{CD}$, celiac disease. 
subject samples per analyte run in replicates of 16 on each of five plates. The cumulative total of the 16 replicates across the five plates ( $n=80$ repeat measurements) was used to determine the inter-assay (between-run) precision. Inter-assay precision included variables for days $(n=3)$, operators $(n=2)$, lots of reagents $(n=2)$, sqidlite platforms $(n=3)$ and assay sites $(n=2)$.

\section{Diagnostic accuracy}

The cut-off value indicates the criterion interval that predicts a positive condition. Screening cut-off values were determined in the pooled CD population by receiver operator curve (ROC) analysis to maximize sensitivity, specificity, Youden index and positive LR+. Interval cut-off values were calculated at maximum specificity. Method comparison studies for the Ig_plex Celiac Disease Panel reported \% overall agreement. Indeterminate values (IND) within \pm 2 standard deviations of the cut-off were removed from the analyses of $n=49$ matrix equivalence samples and $n=70$ positive and negative samples tested in both the ELISA and Ig_plex assays. Matrix equivalence studies reported \% overall agreement and correlation coefficient.

The following definitions and computations of Bayesian diagnostic parameters were used to compare the different $\mathrm{CD}$ populations in the microarray based on standard $2 \times 2$ concordance table analysis with $\mathrm{A}=$ true positives, $\mathrm{B}=$ false positives, $\mathrm{C}=$ false negatives and $\mathrm{D}=$ true negatives:

- $\quad$ TPR is the true positive rate or sensitivity $=A /(A+C)$.

- $\quad$ TNR is the true negative rate or specificity $=D /(B+D)$.

- $\quad$ PPV is the positive predictive value or precision $=A /(A+B)$.

- Accuracy (overall agreement) is the proportion correctly classified $=(A+D) /(A+B+C+D)$.

- Youden index is the sensitivity + specificity -1 .

- $\quad$ LR+ is the positive likelihood ratio, or the ratio of TPR (sensitivity) to FPR $(1$ - specificity). $(\mathrm{A} /(\mathrm{A}+\mathrm{C})) /(\mathrm{B} /(\mathrm{B}+\mathrm{D}))$.

- Post-test probability (p) is the adjusted PPV based on the known prevalence of $\mathrm{CD}$ (pretest probability $=1 \%$ ).
Calculations and ROC analyses were conducted using MedCalc (version 16.4.3) and NCSS12 (version 12.0.10).

\section{Ig_plex Celiac Disease Panel multiple test likelihood ratios}

To investigate the clinical utility of multiplexing, combined specificity measures and multiple LR+ calculations were determined based on combinations of single test clinical performance indices. Computing post-test odds after a series of diagnostic tests is more informative than using the single test sensitivity/specificity method. When using multiple tests, the probabilities are converted to odds ratios (OR) then back to probabilities: odds = probability/ (1 - probability). For multiplexed tests, post-test odds $=$ pretest odds $\times$ LR $1 \times$ LR $2 \times$ LR $3 \ldots \times$ LRn for discreet positive tests. Post-test odds are then converted back to a probability by the following: probability $=$ odds $/(1+$ odds $)$ [33].

\section{Results}

\section{Analytical performance of the Ig_plex Celiac Disease Panel}

The analytical precision results of the Ig_plex Celiac Disease Panel are reported in Table 3. Intra-assay precision of each of the five plates was $\leq 15 \%$ CV for all PC plasma samples and antibodies tested. Inter-assay precision across all five plates was $\leq 15 \% \mathrm{CV}$ for each PC and antibody.

Five plates were run over 3 days by two operators, on three sqidlite platforms across two testing sites with PC

Table 3: Ig_plex Celiac Disease Panel precision performance.

\begin{tabular}{|c|c|c|c|c|c|c|c|c|c|}
\hline \multirow[t]{2}{*}{ Sample } & \multirow[t]{2}{*}{ Plate } & \multicolumn{2}{|c|}{$\begin{array}{r}\text { tTG IgA intra-assay } \\
(n=16)\end{array}$} & \multicolumn{2}{|c|}{$\begin{array}{r}\text { tTG IgG intra-assay } \\
(n=16)\end{array}$} & \multicolumn{2}{|c|}{$\begin{array}{r}\text { DGP IgA intra-assay } \\
(n=16)\end{array}$} & \multicolumn{2}{|c|}{$\begin{array}{l}\text { DGP IgG intra-assay } \\
\qquad(n=16)\end{array}$} \\
\hline & & Avg U/mL & $\% \mathrm{CV}$ & Avg U/mL & $\% \mathrm{CV}$ & Avg U/mL & $\% \mathrm{CV}$ & $\operatorname{Avg~U/mL~}$ & $\% \mathrm{CV}$ \\
\hline \multirow[t]{5}{*}{ PC1 } & 1 & 109.1 & 6.4 & 53.5 & 7.4 & 80.0 & 10.8 & 95.1 & 9.7 \\
\hline & 2 & 110.1 & 8.9 & 67.0 & 8.2 & 80.1 & 7.3 & 102.4 & 8.1 \\
\hline & 3 & 126.0 & 13.5 & 57.1 & 7.0 & 82.1 & 10.9 & 113.5 & 6.9 \\
\hline & 4 & 100.9 & 4.8 & 58.9 & 9.8 & 79.0 & 8.0 & 98.4 & 6.7 \\
\hline & 5 & 125.8 & 9.7 & 66.2 & 9.2 & 79.4 & 9.6 & 96.7 & 14.8 \\
\hline \multicolumn{2}{|c|}{ Inter-assay $(\mathrm{n}=80)$} & 114.4 & 12.8 & 60.6 & 12.0 & 80.1 & 9.3 & 101.2 & 11.4 \\
\hline \multirow[t]{5}{*}{ PC2 } & 1 & 54.0 & 10.2 & 24.9 & 6.0 & 34.1 & 8.3 & 29.2 & 10.1 \\
\hline & 2 & 56.6 & 6.4 & 30.3 & 10.6 & 37.2 & 8.4 & 31.7 & 9.0 \\
\hline & 3 & 56.4 & 10.9 & 29.3 & 5.8 & 38.3 & 11.8 & 36.3 & 11.9 \\
\hline & 4 & 52.9 & 6.6 & 28.7 & 7.6 & 36.2 & 8.0 & 30.8 & 8.5 \\
\hline & 5 & 59.2 & 8.5 & 29.5 & 8.0 & 36.3 & 11.2 & 27.7 & 9.1 \\
\hline \multicolumn{2}{|c|}{ Inter-assay $(\mathrm{n}=80)$} & 55.8 & 9.4 & 28.5 & 10.3 & 36.4 & 10.3 & 31.1 & 13.6 \\
\hline
\end{tabular}

CV, coefficient of variation; DGP, deamidated gliadin peptide; PC, positive control; tTG, tissue transglutaminase. 
assayed in replicates of 16 on each plate for intra-assay precision $\% \mathrm{CV}$. A total of 80 results across the five plates were obtained for inter-assay precision $\% \mathrm{CV}$.

\section{Ig_plex Celiac Disease Panel cut-off determination}

To establish the screening cut-off value for the assay, clinically defined CD samples $(n=143)$ were compared to the normal samples $(n=61)$. The cut-off values that maximized sensitivity, specificity, Youden index and positive LR were selected (Table 4). The screening cut-off was then used for method comparison and matrix equivalence accuracy studies. In addition, 20 WA samples were tested as a related disease control group and compared to the CD population. None of the WA samples demonstrated antibodies to tTG or DGP IgG and IgA isotypes.

\section{Method comparison to ELISA}

Agreement (accuracy) of the Ig_plex Celiac Disease Panel to FDA-approved ELISA kits was conducted by standard $2 \times 2$ concordance table analysis and reported in Table 5 . The method comparison study for the Ig_plex Celiac Disease Panel used the screening cut-offs to evaluate $\mathrm{n}=70$ CD subjects.

\section{Matrix equivalence}

To extend the sample matrix use of the Ig_plex Celiac Disease Panel, matrix equivalence studies were sequentially performed using matched normal and CD samples to measure agreement between 1) serum and venous plasma and 2) venous and capillary plasma.

\section{Serum to plasma equivalence}

To validate the use of plasma as the sample matrix, equivalence testing was conducted on matched serum and plasma samples drawn consecutively from $34 \mathrm{CD}$ subjects without biopsy confirmation, five WA subjects and 10 healthy donors (Table 2). Agreement analysis of observed results between both matrices is summarized in Table 6.

\section{Venous to capillary plasma equivalence}

To verify that capillary EDTA plasma was also a reliable matrix for the Ig_plex Celiac Disease Panel, paired venous and finger-prick blood (Microvette 100 EDTA) draws ( $n=23$, Table 2) were commissioned at commercial enrollment sites. Agreement $(\mathrm{n}=21+2$ IND) and correlation analyses were then conducted on the pooled results (Table 7, Figure 1).

Table 4: Clinical performance features for selecting screening cut-off values for CD serum and plasma samples.

\begin{tabular}{lrrrrrrr}
\hline Antibody & $\begin{array}{r}\text { Cutoff } \\
(\mathbf{U} / \mathrm{mL})\end{array}$ & $\begin{array}{r}\text { TPR } \\
\text { (Sens.) }\end{array}$ & $\begin{array}{r} \pm \mathbf{9 5 \%} \\
\mathrm{Cl}\end{array}$ & $\begin{array}{r}\text { TNR } \\
\text { (Spec.) }\end{array}$ & $\begin{array}{r} \pm \mathbf{9 5 \%} \\
\mathbf{C l}\end{array}$ & $\begin{array}{r}\text { Youden } \\
\text { Index }\end{array}$ & $\begin{array}{r}\text { Max } \\
\text { LR+ }\end{array}$ \\
\hline tTG IgA & $\geq 5.6$ & 0.96 & $0.91-0.98$ & 0.98 & $0.91-0.99$ & 0.94 & 58.4 \\
tTG IgG & $\geq 6.4$ & 0.89 & $0.83-0.94$ & 0.98 & $0.91-0.99$ & 0.87 & 54.2 \\
DGP IgA & $\geq 14.7$ & 0.82 & $0.70-0.91$ & 0.93 & $0.84-0.98$ & 0.75 & 12.5 \\
DGP IgG & $\geq 10.5$ & 0.83 & $0.72-0.92$ & 0.90 & $0.79-0.97$ & 0.73 & 8.4 \\
\hline
\end{tabular}

$\mathrm{Cl}$, confidence interval; DGP, deamidated gliadin peptide; LR, likelihood ratio; TNR, true negative rate; TPR, true positive rate; tTG, tissue transglutaminase.

Table 5: Agreement of Ig_plex Celiac Disease Panel multiplexed results to single ELISA results.

\begin{tabular}{lrrrr}
\hline Agreement & tTG IgA & tTG IgG & DGP IgA & DGP IgG \\
\hline Positive agreement & $98 \%(46 / 47)$ & $100 \%(29 / 29)$ & $97 \%(37 / 38)$ & $88 \%(32 / 36)$ \\
Negative agreement & $100 \%(23 / 23)$ & $82 \%(32 / 39)$ & $87 \%(27 / 31)$ & $91 \%(29 / 32)$ \\
Overall agreement & $99 \%(69 / 70)$ & $90 \%(61 / 68)$ & $93 \%(64 / 69)$ & $90 \%(61 / 68)$ \\
\hline
\end{tabular}

DGP, deamidated gliadin peptide; tTG, tissue transglutaminase. 
Table 6: Serum to plasma agreement analysis results for Ig_Plex Celiac Disease Panel.

\begin{tabular}{lrrrr}
\hline Agreement & tTG IgG & DGP IgG & tTG IgA & DGP IgA \\
\hline Positive agreement & $100 \%(8 / 8)$ & $100 \%(12 / 12)$ & $91 \%(10 / 11)$ & $100 \%(10 / 10)$ \\
Negative agreement & $100 \%(41 / 41)$ & $97 \%(36 / 37)$ & $100 \%(38 / 38)$ & $100 \%(39 / 39)$ \\
Overall agreement & $100 \%(49 / 49)$ & $98 \%(48 / 49)$ & $98 \%(48 / 49)$ & $100 \%(49 / 49)$ \\
\hline
\end{tabular}

DGP, deamidated gliadin peptide; tTG, tissue transglutaminase.

Table 7: Venous to capillary (finger prick) plasma agreement analysis results for Ig_Plex Celiac Disease Panel.

\begin{tabular}{lrrrr}
\hline Agreement & tTG IgG & DGP IgG & tTG IgA & DGP IgA \\
\hline Positive agreement & $100 \%(2 / 2)$ & $100 \%(5 / 5)$ & $100 \%(3 / 3)$ & $100 \%(3 / 3)$ \\
Negative agreement & $100 \%(19 / 19)$ & $100 \%(16 / 16)$ & $100 \%(18 / 18)$ & $100 \%(18 / 18)$ \\
Overall agreement & $100 \%(21 / 21)$ & $100 \%(21 / 21)$ & $100 \%(21 / 21)$ & $100 \%(21 / 21)$ \\
\hline
\end{tabular}

DGP, deamidated gliadin peptide; tTG, tissue transglutaminase.

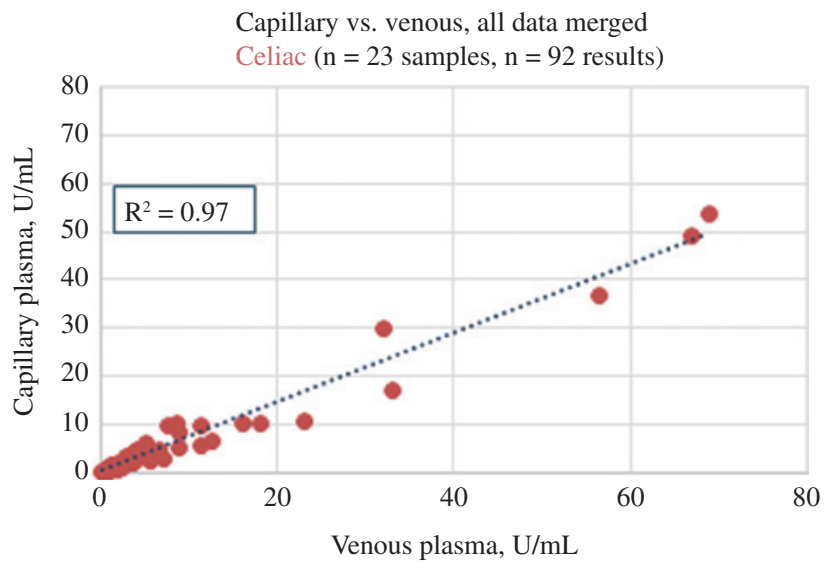

Figure 1: Combined normal and celiac capillary vs. venous plasma sample correlation, $\mathrm{R}^{2}$.

\section{Multiple likelihood ratios of the Ig_plex Celiac Disease Panel}

Multiple LR+ calculations can aid in ruling-in the diagnosis of CD when screening suspected cases or the general population [18, 20]. Increased clinical utility of the Ig_plex Celiac Disease Panel's multiplexing capabilities is further exemplified using the multiple LR+ approach [33]. To calculate the multiple $\mathrm{LR}+$ from several positive test results, the pretest OR is first calculated with CD prevalence $=0.01$. Using the LR+ values obtained from the screening cutoff, LR+ combinations in multi-reactive samples yielded a much higher post-test probability of having $\mathrm{CD}$, especially if one of the positive tests were for tTG (IgA or IgG). Samples positive for both DGP IgA and IgG antibodies also increase the probability of disease in all test combinations, even in the absence of tTG positivity (Table 8).

\section{Diagnostic accuracy of the Ig_plex Celiac Disease Panel}

Diagnostic accuracy evaluation was performed on subjects stratified as Marsh III biopsy-confirmed CD with and without GFD treatment and CD without Marsh III biopsy confirmation that includes potential and classical CD.

Table 8: Multiple test positivity increases post-test probability of CeD.

\begin{tabular}{|c|c|c|c|c|c|c|c|c|c|c|c|}
\hline \multirow[t]{3}{*}{ Antibody } & \multirow[t]{3}{*}{ LR+ } & \multirow{3}{*}{$\begin{array}{l}\text { One test } \\
\text { positive }\end{array}$} & & & & & \multicolumn{5}{|c|}{ Post-test probability for CeD, \% } \\
\hline & & & \multicolumn{4}{|c|}{ Two tests positive } & \multicolumn{4}{|c|}{ Three tests positive } & \multirow{2}{*}{$\begin{array}{r}\text { Four tests } \\
\text { positive }\end{array}$} \\
\hline & & & $\begin{array}{l}\text { tTG IgA } \\
\text { tTG IgG }\end{array}$ & $\begin{array}{r}\text { tTG IgA } \\
\text { DGP IgA }\end{array}$ & $\begin{array}{l}\text { tTG IgG } \\
\text { DGP IgG }\end{array}$ & $\begin{array}{l}\text { DGP IgA } \\
\text { DGP IgG }\end{array}$ & $\begin{array}{l}\text { tTG IgA DGP } \\
\text { IgA DGP IgG }\end{array}$ & $\begin{array}{r}\text { tTG IgA tTG } \\
\text { IgG DGP IgG }\end{array}$ & $\begin{array}{l}\text { tTG IgA tTG } \\
\text { IgG DGP IgA }\end{array}$ & $\begin{array}{l}\text { tTG IgG DGP } \\
\text { IgG DGP IgA }\end{array}$ & \\
\hline tTG IgA & 58.4 & 37.0 & 96.9 & 88.0 & & & 98.4 & 99.6 & 99.7 & & 100.0 \\
\hline tTG IgG & 54.2 & 35.0 & 96.9 & & 82.0 & & & 99.6 & 99.7 & 98.3 & 100.0 \\
\hline DGP IgA & 12.5 & 11.0 & & 88.0 & & 51.2 & 98.4 & & 99.7 & 98.3 & 100.0 \\
\hline DGP IgG & 8.4 & 9.0 & & & 82.0 & 51.2 & 98.4 & 99.6 & & 98.3 & 100.0 \\
\hline
\end{tabular}

CD, celiac disease; DGP, deamidated gliadin peptide; LR, likelihood ratio; tTG, tissue transglutaminase. 
Both serum and plasma matrices were available for the CD population without biopsy confirmation to further validate the use of plasma as a sample type.

\section{Multiplexing and response to GFD treatment}

Rank correlation analyses were conducted to examine if tTG or DGP antibody positivity in terms of measured $\mathrm{U} / \mathrm{mL}$ were correlated to GFD treatment duration. Treatment time was available for $n=40$ of the confirmed CD with GFD group and ranged from 1 to 184 weeks (median $=20$ weeks). No significant Spearman's rank correlation coefficient was found for any antibody in terms of GFD treatment time within this group. Thus, high and low positive values were equally distributed over the entire treatment time.

Diagnostic accuracy results for the treated and untreated biopsy-confirmed CD populations are presented in Table 9. At the screening cut-off value, tTG IgA antibody detection in confirmed CD without GFD intervention sensitivity and specificity were $0.97(95 \% \mathrm{CI}=0.83-0.99)$ and 0.98 (0.91-0.99), respectively. GFD treatment as a whole, did not reduce the sensitivity and specificity of tTG IgA detection. For tTG IgG antibodies, the sensitivity and specificity for confirmed CD without GFD treatment were 0.97 (0.83-0.99) and 0.98 (0.91-0.99), respectively. In this subpopulation, almost all cases (30/31) had both tTG IgA and IgG. At the screening cut-off, GFD treatment had little impact on the sensitivity or diagnostic accuracy.

DGP IgA clinical performance in Marsh III biopsyconfirmed CD without GFD treatment, gave a sensitivity of $0.97(0.83-0.99)$ and specificity of $0.89(0.83-0.94)$ at the screening cut-off. A decrease to $0.90(0.80-0.96)$

Table 9: Diagnostic accuracy metrics for the biopsy-confirmed CD populations.

\begin{tabular}{lcccccccc}
\hline Antibody & Biopsy GFD & $\begin{array}{r}\text { TPR } \\
\text { (Sens.) }\end{array}$ & $\begin{array}{r}\text { TNR Accuracy } \\
\text { (Spec.) }\end{array}$ & PPV & NPV & LR+ \\
\hline tTG IgA & + & + & 0.97 & 0.98 & 0.98 & 0.97 & 0.98 & 59.0 \\
& + & - & 0.98 & 0.98 & 0.98 & 0.98 & 0.98 & 59.8 \\
tTG IgG & + & + & 0.97 & 0.98 & 0.98 & 0.97 & 0.98 & 59.0 \\
& + & - & 0.90 & 0.98 & 0.95 & 0.98 & 0.92 & 55.1 \\
DGP IgA & + & + & 0.97 & 0.89 & 0.91 & 0.81 & 0.98 & 8.4 \\
& + & - & 0.90 & 0.89 & 0.89 & 0.87 & 0.92 & 7.9 \\
DGP IgG & + & + & 0.97 & 0.98 & 0.98 & 0.97 & 0.98 & 59.0 \\
& + & - & 0.75 & 0.98 & 0.87 & 0.97 & 0.81 & 44.6 \\
\hline
\end{tabular}

DGP, deamidated gliadin peptide; GFD, gluten-free diet; LR, likelihood ratio; NPV, negative predictive value; PPV, positive predictive value; TNR, true negative rate; TPR, true positive rate; tTG, tissue transglutaminase. sensitivity was observed in response to GFD treatment. At the screening cut-off, DGP IgG detection in biopsyconfirmed CD without GFD had a high sensitivity of 0.97 (0.83-0.99) and specificity of 0.98 (0.91-0.99). In response to GFD treatment, this sensitivity decreased significantly approaching a $\mathrm{p} \leq 0.05$ level (two-sided t-test $\mathrm{p}=0.07$ ) to $0.75(0.63-0.86)$.

\section{Positive test intervals and response to GFD treatment}

Based on the drop-in sensitivity for DGP IgG in response to GFD treatment, we investigated whether increasing the test interval to stratify high positives and low positives [23] would better partition GFD treatment response. ROC analyses of biopsy-confirmed CD indicated that if the screening cut-off interval reported in Table 4 was increased to the threshold interval reported in Table 10, a significant drop in sensitivity was found in response to GFD treatment for two of the analytes. This is interpreted to mean that at higher test interval, fewer high positives are seen in the confirmed CD with the GFD treatment group than in the confirmed CD without GFD treatment. For tTG IgA the decrease was $15.9 \%$, for $\mathrm{tTG}$ IgG, $45.7 \%$, for DGP IgA, the decrease was $23.5 \%$ and for DGP IgG, $35.8 \%$. Significance was calculated with a two-sided t-test assuming equal variance in the two $\mathrm{CD}$ populations.

\section{Diagnostic accuracy in CD serum and plasma}

To further evaluate the use of plasma matrix to report $C D$ results, both serum and plasma from subjects without Marsh III biopsy confirmation inclusive of potential or

Table 10: GFD treatment response as a function of test interval.

\begin{tabular}{|c|c|c|c|c|c|c|}
\hline \multirow[t]{2}{*}{ Antibody } & \multirow{2}{*}{$\begin{array}{l}\text { Confirmed } \\
\text { CeD }\end{array}$} & \multirow{2}{*}{$\begin{array}{r}\text { Cut-off } \\
\text { interval }\end{array}$} & \multirow{2}{*}{$\begin{array}{r}\text { Sens. } \\
\text { TPR }\end{array}$} & \multicolumn{2}{|r|}{$95 \% \mathrm{Cl}$} & \multirow{2}{*}{$\begin{array}{r}\text { Significance, } \\
\text { p }\end{array}$} \\
\hline & & & & Lower & Upper & \\
\hline \multirow[t]{2}{*}{ tTG IgA } & No GFD & $\geq 34.9$ & 0.94 & 0.79 & 0.99 & 0.190 \\
\hline & $+\mathrm{GFD}$ & $\geq 34.7$ & 0.79 & 0.65 & 0.89 & \\
\hline \multirow[t]{2}{*}{ tTG IgG } & No GFD & $\geq 29.6$ & 0.81 & 0.63 & 0.93 & $0.002^{\mathrm{a}}$ \\
\hline & $+\mathrm{GFD}$ & $\geq 30.3$ & 0.44 & 0.30 & 0.59 & \\
\hline \multirow[t]{2}{*}{ DGP IgA } & No GFD & $\geq 32.8$ & 0.81 & 0.63 & 0.93 & 0.097 \\
\hline & $+\mathrm{GFD}$ & $\geq 32.0$ & 0.62 & 0.47 & 0.75 & \\
\hline \multirow[t]{2}{*}{ DGP IgG } & No GFD & $\geq 29.2$ & 0.81 & 0.63 & 0.93 & $0.012^{\mathrm{a}}$ \\
\hline & $+\mathrm{GFD}$ & $\geq 29.1$ & 0.52 & 0.38 & 0.66 & \\
\hline
\end{tabular}

aSignificant $\mathrm{p} \leq 0.05 . \mathrm{CD}$, celiac disease; $\mathrm{Cl}$, confidence interval; DGP, deamidated gliadin peptide; GFD, gluten-free diet; TPR, true positive rate; tTG, tissue transglutaminase. 
Table 11: Diagnostic accuracy metrics for CD without biopsy confirmation serum and plasma samples.

\begin{tabular}{lrrrrrrr}
\hline Antibody & Matrix & $\begin{array}{r}\text { TPR } \\
\text { (Sens.) }\end{array}$ & $\begin{array}{r}\text { TNR } \\
\text { (Spec.) }\end{array}$ & Accuracy & PPV & NPV & LR+ \\
\hline tTG IgA & Serum & 1.00 & 0.98 & 0.99 & 0.95 & 1.00 & 61.0 \\
& Plasma & 0.90 & 0.98 & 0.95 & 0.97 & 0.94 & 54.9 \\
tTG IgG & Serum & 0.90 & 0.98 & 0.96 & 0.95 & 0.97 & 54.9 \\
& Plasma & 0.88 & 0.98 & 0.94 & 0.97 & 0.92 & 53.4 \\
DGP IgA & Serum & 0.95 & 0.92 & 0.93 & 0.79 & 0.98 & 11.6 \\
& Plasma & 0.90 & 0.87 & 0.88 & 0.82 & 0.93 & 6.9 \\
DGP IgG & Serum & 0.75 & 0.97 & 0.91 & 0.88 & 0.92 & 22.9 \\
& Plasma & 0.83 & 0.95 & 0.90 & 0.92 & 0.89 & 16.8 \\
\hline
\end{tabular}

DGP, deamidated gliadin peptide; LR, likelihood ratio; NPV, negative predictive value; PPV, positive predictive value; TNR, true negative rate; TPR, true positive rate; $\mathrm{tTG}$, tissue transglutaminase.

classical CD were collected $[1,28]$. Both serum and plasma samples from these subjects showed similar sensitivity, specificity and accuracy for all analytes (Table 11). Modest, non-significant decreases in plasma DGP IgA sensitivity and accuracy were seen compared to serum.

\section{CD sample multi-reactivity and disease progression}

At the screening cut-off, samples were found to decrease in the frequency of multiple positive test results when comparing Marsh III biopsy-confirmed CD to the CD population without Marsh III biopsy confirmation. Interestingly, the number of samples positive for all four antibodies decreased from $93.5 \%$ in biopsy-confirmed CD to $55.0 \%$ in sera from CD without confirmation and to $47.5 \%$ in CD without confirmation plasma. For biopsy-confirmed CD without GFD, $28 / 31(90.3 \%)$ of samples had all four antibodies positive, 2/31 (6.5\%) samples demonstrated three positive results and one subject two positive results. In the cases of $\mathrm{CD}$ without confirmation serum, multi-reactivity was reduced in frequency to: $11 / 20$ (55.0\%) for four positives; $6 / 20(30.0 \%)$ for three positives; $2 / 20(10.0 \%)$ for two positives; $1 / 20$ (5.0\%) for one positive. In CD without confirmation plasma, 19/40 (47.5\%) were positive for all four antibodies; $7 / 40$ (17.5\%) for three positives; $9 / 40$ (22.5\%) for two positives; $4 / 40$ (10.0\%) for one positive and one sample no positive result.

\section{Discussion}

The precise Ig_plex Celiac Disease Panel was used to evaluate clinical performance metrics when testing all four relevant antibody $C D$ markers in a multiplexed panel: tTG IgA, tTG IgG, DGP IgA and DGP IgG. The sensitivity, specificity, diagnostic accuracy, LR and post-test probabilities of disease were compared in CD populations with and without Marsh III biopsy confirmation and the clinical utility of using venous and capillary plasma assessed.

Sensitivity and specificity of serum tTG IgA is typically high (e.g. 94.3\% and 95.7\%) with a PPV of 95.7\%. The Ig_plex Celiac Disease Panel demonstrated equivalent or better performance for tTG IgA as that reported for standard ELISA in the literature $[15,19,34,35]$. In the group without biopsy confirmation, high sensitivity and specificity was preserved indicating that TTG IgA is an accurate screening marker in both serum (0.99) and plasma (0.96). Ig_plex detection of tTG IgG also showed high sensitivity (0.97) and specificity (0.98) in confirmed CD with a diagnostic accuracy of 0.98. For screening tTG IgG in plasma, the accuracy was 0.93 . These values are comparable to other high sensitivity (up to 0.95 ) and specificity (up to 1.0) tTG IgG kits reported in the literature which typically use recombinant tTG [36].

Compared to confirmed CD without GFD treatment, both plasma DGP IgA and IgG demonstrated modest decreases in sensitivity and accuracy in $\mathrm{CD}$ without confirmation. However, when DGP positive results were combined with other test results like tTG IgA, the combined tests increased the post-test probability of ruling-in $\mathrm{CD}$ in plasma beyond that seen for tTG IgA alone. Further, 10\% of CD without biopsy confirmation samples did not have measurable tTG IgA or IgG but did have DGP IgA or IgG antibodies in five of the six observed cases. This demonstrates the power of the Ig_plex Celiac Disease Panel multiplex test, that when multiple LR+ values are determined including those for DGP in the absence of tTG positivity, there is a greater probability of screening for and ruling-in $\mathrm{CD}$. When three to four tests are positive, there is over $95 \%$ probability the subject will have CD. A detailed examination of multiple LR+ probabilities and the percentage of multi-reactive test results for the four positive test combinations promote the diagnosis of $\mathrm{CD}$ in a screening population and allows for more marker-oriented monitoring and management of patients.

Similar to the results reported here, Vermeersch et al. $[20,23]$ found that LR+ for CD markedly increased with double positivity and increasing antibody levels of IgA anti-tTG and IgG anti-DGP. Subjects with double positivity and high antibody levels had a high probability for having CD. The detected fraction of CD subjects with double positivity and high antibody levels was reported as $59-67 \%$. Our results extend this observation to all 
four autoantibody markers where a distinct pattern of multi-reactivity was observed in the different CD populations. In CD samples without confirmation, $47-67 \%$ of subject samples had all four antibodies positive. In the current study, antibody multi-reactivity increased in the confirmed $\mathrm{CD}$ group when compared to the $\mathrm{CD}$ without confirmation group.

Leffler et al. suggested that if more than one celiac serologic test is positive, it strengthens the argument that the subject has a true, if mild, form of $\mathrm{CD}$ when examining potential CD subjects [37]. Dahlbom et al. concluded that detection of IgA/IgG tTG has the potential of detecting most untreated CD patients, including those with IgA deficiency, which supports the use of multiple LR+ combinations for more than one positive test to rule-in CD [21]. Although total IgA was not herein tested, the use of the four Ig_plex antibodies reduces the occurrence of false negatives as shown by the multiple LR+ values.

Raising the cut-off for a positive result to the threshold interval above the screening cut-off significantly reduced the sensitivity of tTG IgG in the confirmed CD group with GFD treatment by $46 \%$ compared to just a $16 \%$ reduction in confirmed active CD without GFD. Thus, the degree of positivity or relative affinity for $\mathrm{TTG}$ IgG antibodies were reduced in response to GFD treatment in the subject samples evaluated here. When the DGP IgA cut-off was raised, sensitivity dropped $23.5 \%$ in the GFD treatment group. For DGP IgG, the sensitivity dropped significantly by $35.8 \%$ at almost 3-fold elevated cut-off when comparing confirmed active CD without GFD to confirmed CD with GFD. This threshold analysis is important in the determination of how positive test levels change with respect to treatment. Within the GFD treatment group there is no correlation of treatment time and antibody positive level. However, there is a significant relation of treatment to degree of antibody positivity when comparing to the confirmed CD without any GFD treatment group. While likely, prospective studies need to demonstrate whether tTG IgG and DGP IgG alone or in combination with the other multiplex analytes are sensitive indicators of compliance to GFD treatment.

In one study, DGP IgA (sensitivity 87.4, specificity 97.2) and the combination of DGP IgA + IgG (sensitivity 94.7, specificity 89.6) were able to monitor compliance to GFD in CD children [38]. It was concluded that both antiDGPs showed higher sensitivity than TTG IgA in monitoring compliance with GFD but did not out-perform tTG IgA for $\mathrm{CD}$ screening. These findings agree in part with those reported here, with the Ig_plex Celiac Disease Panel tTG IgG test result providing additional benefit. A published guideline recommended that for monitoring adherence to GFD, serological testing of IgA tTG or IgA (or IgG) DGP antibodies should be conceived [10].

In addition, confirmed CD without GFD had high sensitivity for both DGP IgA and IgG (0.97 each). At the optimal screening cut-off point, DGP IgG sensitivity decreased to 0.75 in the CD group lacking Marsh III biopsy confirmation. Given the differential changes in antibody positivity and multi-reactivity detected by the Ig_plex Celiac Disease Panel in different CD populations, this multiplex assay could prove useful in patient management such as monitoring disease prevalence and progression in view of the iceberg paradigm of $\mathrm{CD}$. Correlation of our multi-reactive antibody profiles to symptoms and biopsy results, which has been performed for tTG IgA only, awaits further study [14, 20, 39-41].

Altogether, the diagnostic efficiency of the Ig_plex Celiac Disease Panel was demonstrated in four ways: (1) plasma was as clinically accurate as serum for use as a sample matrix; (2) multi-reactive test results obtained from the Ig_plex Celiac Disease Panel increase the probability of ruling in $\mathrm{CD}$ when screening the general population; (3) multiplexing the known $\mathrm{CD}$ antibodies might facilitate effective GFD treatment monitoring and (4) multiplexing all four tTG and DGP antibodies might aid the ability to monitor the progression of $\mathrm{CD}$.

As a control group, those with WA had no celiac antibody reactivity. Although this multiplex platform will not support or rule out a diagnosis of NCGS/NCWS, a condition for which reliable biomarkers for diagnosis are lacking [42], when Wheat IgE testing along with CD testing results are negative (thus also making WA less likely, as WA is most often IgE-mediated), the health care provider may want to discuss the possibility of NCGS/NCWS with the patient. Adding other known wheat extract/gluten allergens like fermentable oligo-, di-, mono-saccharides and polyols or wheat amylase trypsin inhibitors or other cereal prolamins to the microarray might potentially facilitate its use in screening for WA subtypes, NCGS/NCWS and other intolerances to grains [8, 42-47].

Furthermore, this study demonstrates that venous plasma is a diagnostically accurate sample matrix and that the use of finger-prick capillary plasma is also viable for evaluating potential CD antibodies based on agreement and correlation to venous plasma. In a screening study of 198 children using capillary blood plasma, $13.6 \%$ tested positive for ITG IgA, tTG IgG or gliadin IgA antibodies in a rapid immunochromatographic test [26]. In another study, 31 of 41 children who screened positive for tTG IgA or IgG using finger-prick capillary plasma, were confirmed positive on a serum-based endomysial antibody test [27]. If screening undiagnosed or self-diagnosed GRDs is 
facilitated by using finger-prick collection methods, with multiplexed quantitative results obtained in a clinical laboratory, then a physician-guided use of GFD treatment can be performed with improved treatment and disease monitoring [3, 10, 11, 14-17, 48]. Additional prospective case finding and follow up studies in large populations using capillary plasma for our Ig_plex Celiac Disease Panel are underway.

Acknowledgments: The authors wish to acknowledge Dr. Eleftherios Diamandis for his critical review of the manuscript.

Author contributions: All the authors have accepted responsibility for the entire content of this submitted manuscript and approved submission.

Research funding: Sources of support: Internally funded. There was no involvement of a pharmaceutical company.

Employment or leadership: None declared.

Honorarium: None declared.

Competing interests: The funding organization(s) played no role in the study design; in the collection, analysis, and interpretation of data; in the writing of the report; or in the decision to submit the report for publication.

\section{References}

1. Ludvigsson JF, Leffler DA, Bai JC, Biagi F, Fasano A, Green PH, et al. The Oslo definitions for coeliac disease and related terms. Gut 2013;62:43-52.

2. Catassi C, Alaedini A, Bojarski C, Bonaz B, Bouma G, Carroccio A, et al. The overlapping area of non-celiac gluten sensitivity (NCGS) and wheat-sensitive irritable bowel syndrome (IBS): an update. Nutrients 2017;9:1268.

3. Guandalini S, Polanco I. Nonceliac gluten sensitivity or wheat intolerance syndrome? J Pediatrics 2015;166:805-11.

4. Ludvigsson JF, Ciacci C, Green PH, Kaukinen K, Korponay-Szabo IR, Kurppa K, et al. Outcome measures in coeliac disease trials: the Tampere recommendations. Gut 2018;67:1410-24.

5. Choung RS, Larson SA, Khaleghi S, Rubio-Tapia A, Ovsyannikova IG, King KS, et al. Prevalence and morbidity of undiagnosed celiac disease from a community-based study. Gastroenterology 2017;152:830-9.

6. Elli L, Branchi F, Tomba C, Villalta D, Norsa L, Ferretti F, et al. Diagnosis of gluten related disorders: celiac disease, wheat allergy and non-celiac gluten sensitivity. World J Gastroenterol 2015;21:7110-9.

7. Sicherer SH, Allen K, Lack G, Taylor SL, Donovan SM, Oria M. Critical issues in food allergy: a national academies consensus report. Pediatrics 2017;140:e20170194.

8. Fasano A, Sapone A, Zevallos V, Schuppan D. Nonceliac gluten sensitivity. Gastroenterology 2015;148:1195-204.

9. Husby S, Koletzko S, Korponay-Szabó IR, Mearin ML, Phillips A, Shamir R, et al. European Society for Pediatric Gastroenterology,
Hepatology, and Nutrition guidelines for the diagnosis of coeliac disease. J Pediatr Gastroenterol Nutr 2012;54:136-60.

10. Rubio-Tapia A, Hill ID, Kelly CP, Calderwood AH, Murray JA. ACG clinical guidelines: diagnosis and management of celiac disease. Am J Gastroenterol 2013;108:656-76.

11. Elli L, Ferretti F, Orlando S, Vecchi M, Monguzzi E, Roncoroni L, et al. Management of celiac disease in daily clinical practice. Eur J Intern Med 2019;61:15-24.

12. Dieterich W, Ehnis T, Bauer M, Donner P, Volta U, Riecken EO, et al. Identification of tissue transglutaminase as the autoantigen of celiac disease. Nat Med 1997;3:797-801.

13. Schuppan D, Junker Y, Barisani D. Celiac disease: from pathogenesis to novel therapies. Gastroenterology 2009;137: 1912-33.

14. Caja S, Mäki M, Kaukinen K, Lindfors K. Antibodies in celiac disease: implications beyond diagnostics. Cell Mol Immunol 2011;8:103-9.

15. Leffler DA, Schuppan D. Update on serologic testing in celiac disease. Am J Gastroenterol 2010;105:2520-4.

16. Trigoni E, Tsirogianni A, Pipi E, Mantzaris G, Papasteriades C. Celiac disease in adult patients: specific autoantibodies in the diagnosis, monitoring, and screening. Autoimmune Dis 2014;2014:7.

17. Rashid M, Lee J. Serologic testing in celiac disease: practical guide for clinicians. Can Fam Physician 2016;62:38-43.

18. Vermeersch P, Richter T, Hauer AC, Stern M, Uhlig HH, Zimmer $\mathrm{KP}$, et al. Use of likelihood ratios improves clinical interpretation of IgG and IgA anti-DGP antibody testing for celiac disease in adults and children. Clin Biochem 2011;44:248-50.

19. Lakos G, Norman GL, Mahler M, Martis P, Bentow C, Santora D, et al. Analytical and clinical comparison of two fully automated immunoassay systems for the diagnosis of celiac disease. J Immunol Res 2014;2014:371263.

20. Vermeersch P, Geboes K, Mariën G, Hoffman I, Hiele M, Bossuyt $X$. Serological diagnosis of celiac disease: comparative analysis of different strategies. Clin Chim Acta 2012;413:1761-7.

21. Dahlbom I, Nyberg BI, Berntson L, Hansson T. Simultaneous detection of IgA and IgG antibodies against tissue transglutaminase: the preferred pre-biopsy test in childhood celiac disease. Scand J Clin Lab Invest 2016;76:208-16.

22. Sugai E, Moreno ML, Hwang HJ, Cabanne A, Crivelli A, Nachman F, et al. Celiac disease serology in patients with different pretest probabilities: is biopsy avoidable? World J Gastroenterol 2010;16:3144-52.

23. Oyaert M, Vermeersch P, De Hertogh G, Hiele M, Vandeputte $\mathrm{N}$, Hoffman I, et al. Combining antibody tests and taking into account antibody levels improves serologic diagnosis of celiac disease. Clin Chem Lab Med 2015;53:1537-46.

24. Rashid M, Butzner JD, Warren R, Molloy M, Case S, Zarkadas M, et al. Home blood testing for celiac disease: recommendations for management. Can Fam Physician 2009;55:151-3.

25. Korponay-Szabó IR, Szabados K, Pusztai J, Uhrin K, Ludmány E, Nemes E, et al. Population screening for coeliac disease in primary care by district nurses using a rapid antibody test: diagnostic accuracy and feasibility study. Br Med J 2007;335:1244-7.

26. Almazán MV, Ortega E, Moreno Torres R, Tovar M, Romero J, López-Casado MÁ, et al. Diagnostic screening for subclinical celiac disease using a rapid test in children aged $2-4$. Pediatr Res 2015;78:280-5. 
27. Tommasini A, Not T, Kiren V, Baldas V, Santon D, Trevisiol C, et al. Mass screening for coeliac disease using antihuman transglutaminase antibody assay. Arch Dis Child 2004;89:512-5.

28. Ludvigsson JF, Brandt L, Montgomery SM, Granath F, Ekbom A. Validation study of villous atrophy and small intestinal inflammation in Swedish biopsy registers. BMC Gastroenterol 2009;9:19.

29. Cohen JF, Korevaar DA, Altman DG, Bruns DE, Gatsonis CA, Hooft L, et al. STARD 2015 guidelines for reporting diagnostic accuracy studies: explanation and elaboration. BMJ Open 2016;6:e012799.

30. van Gils T, Senler TG, van der Horst HE, Mulder CJJ, Bouma G, de Vries $\mathrm{H}$. The daily practice of (suspected) coeliac disease management by general practitioners: a qualitative approach. Eur J Gen Pract 2018;24:236-42.

31. U.S. Department of Health and Human Services, Food and Drug Administration, Center for Drug Evaluation and Research, Center for Veterinary Medicine. Bioanalytical Method Validation: Guidance for Industry, 2018.

32. Lee H, Lim SY, Kim KH. Validation of the World Health Organization enzyme-linked immunosorbent assay for the quantitation of immunoglobulin $\mathrm{G}$ serotype-specific antipneumococcal antibodies in human serum. J Korean Med Sci 2017;32:1581-7.

33. Parikh R, Parikh S, Arun E, Thomas R. Likelihood ratios: clinical application in day-to-day practice. Indian J Ophthalmol 2009;57:217-21.

34. Health Quality Ontario. Clinical utility of serologic testing for celiac disease in Ontario: an evidence-based analysis. Ont Health Technol Assess Ser 2010;10:1-111.

35. Chou R, Bougatsos C, Blazina I, Mackey K, Grusing S, Selph S. Screening for celiac disease: evidence report and systematic review for the US Preventive Services Task Force. J Am Med Assoc 2017;317:1258-68.

36. Villalta D, Alessio MG, Tampoia M, Tonutti E, Brusca I, Bagnasco $M$, et al. Testing for IgG class antibodies in celiac disease patients with selective IgA deficiency. A comparison of the diagnostic accuracy of 9 IgG anti-tissue transglutaminase, 1 IgG anti-gliadin and 1 IgG anti-deaminated gliadin peptide antibody assays. Clin Chim Acta 2007;382:95-9.
37. Kaswala DH, Veeraraghavan G, Kelly CP, Leffler DA. Celiac disease: diagnostic standards and dilemmas. Diseases 2015;3: 86-101.

38. Monzani A, Rapa A, Fonio P, Tognato E, Panigati L, Oderda G. Use of deamidated gliadin peptide antibodies to monitor diet compliance in childhood celiac disease. J Pediatr Gastroenterol Nutr 2011;53:55-60.

39. Vermeersch P, Geboes K, Mariën G, Hoffman I, Hiele M, Bossuyt $X$. Defining thresholds of antibody levels improves diagnosis of celiac disease. Clin Gastroenterol Hepatol 2013;11:398-403.

40. Brusca I. Overview of biomarkers for diagnosis and monitoring of celiac disease. Adv Clin Chem 2015;68:1-55.

41. Loberman-Nachum N, Schvimer M, Avivi C, Barshack I, Lahad A, Fradkin $A$, et al. Relationships between clinical presentation, serology, histology, and duodenal deposits of tissue transglutaminase antibodies in pediatric celiac disease. Dig Dis 2018;36:369-76.

42. Reese I, Schäfer C, Kleine-Tebbe J, Ahrens B, Bachmann O, Ballmer-Weber B, et al. Non-celiac gluten/wheat sensitivity (NCGS) - a currently undefined disorder without validated diagnostic criteria and of unknown prevalence: position statement of the Task Force on Food Allergy of the German Society of Allergology and Clinical Immunology (DGAKI). Allergo J Int 2018;27:147-51.

43. Cianferoni A. Wheat allergy: diagnosis and management. J Asthma Allergy 2016;9:13-25.

44. Burkhardt JG, Chapa-Rodriguez A, Bahna SL. Gluten sensitivities and the allergist: threshing the grain from the husks. Allergy 2018;73:1359-68.

45. Llanos-Chea A, Fasano A. Gluten and functional abdominal pain disorders in children. Nutrients 2018;10:1491.

46. Zevallos VF, Raker V, Tenzer S, Jimenez-Calvente C, Ashfaq-Khan $M$, Rüssel N, et al. Nutritional wheat amylase-trypsin inhibitors promote intestinal inflammation via activation of myeloid cells. Gastroenterology 2017;152:1100-13.e12.

47. Schuppan D, Pickert G, Ashfaq-Khan M, Zevallos V. Non-celiac wheat sensitivity: differential diagnosis, triggers and implications. Best Pract Res Clin Gastroenterol 2015;29:469-76.

48. Sansotta N, Amirikian K, Guandalini S, Jericho H. Celiac disease symptom resolution: effectiveness of the gluten-free diet. J Pediatr Gastroenterol Nutr 2018;66:48-52. 